Network Working Group

Request for Comments: 3107

Category: Standards Track
Y. Rekhter

Juniper Networks

E. Rosen

Cisco Systems, Inc.

May 2001

\title{
Carrying Label Information in BGP-4
}

Status of this Memo

This document specifies an Internet standards track protocol for the Internet community, and requests discussion and suggestions for improvements. Please refer to the current edition of the "Internet Official Protocol Standards" (STD 1) for the standardization state and status of this protocol. Distribution of this memo is unlimited.

Copyright Notice

Copyright (C) The Internet Society (2001). All Rights Reserved.

Abstract

This document specifies the way in which the label mapping

information for a particular route is piggybacked in the same Border Gateway Protocol (BGP) Update message that is used to distribute the route itself. When BGP is used to distribute a particular route, it can be also be used to distribute a Multiprotocol Label Switching

(MPLS) label which is mapped to that route.

Table of Contents

\begin{tabular}{|c|c|}
\hline 1 & Specification of Requirements \\
\hline 2 & 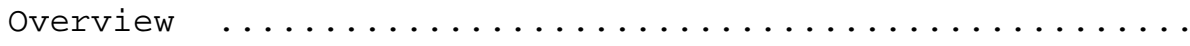 \\
\hline 3 & Carrying Label Mapping Information $\ldots \ldots \ldots \ldots \ldots \ldots \ldots$ \\
\hline 4 & Advertising Multiple Routes to a Destination ......... \\
\hline 5 & 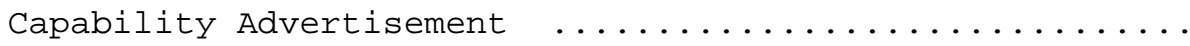 \\
\hline 6 & When the BGP Peers are not Directly Adjacent $\ldots . . . \ldots$ \\
\hline 7 & 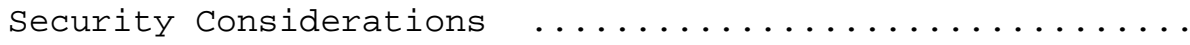 \\
\hline 8 & 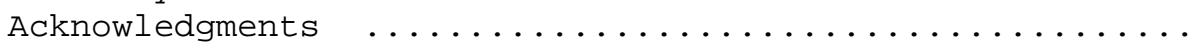 \\
\hline 9 & 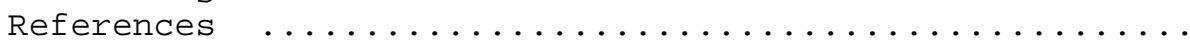 \\
\hline 10 & 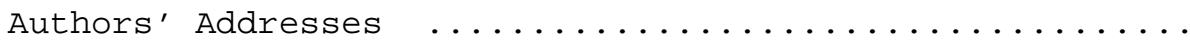 \\
\hline & 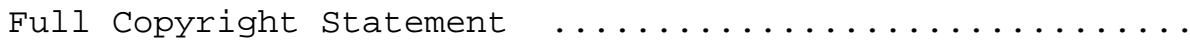 \\
\hline
\end{tabular}




\section{Specification of Requirements}

The key words "MUST", "MUST NOT", "REQUIRED", "SHALL", "SHALL NOT", "SHOULD", "SHOULD NOT", "RECOMMENDED", "MAY", and "OPTIONAL" in this document are to be interpreted as described in RFC 2119.

\section{Overview}

When BGP is used to distribute a particular route, it can also be used to distribute an MPLS label that is mapped to that route [MPLSARCH]. This document specifies the way in which this is done. The label mapping information for a particular route is piggybacked in the same BGP Update message that is used to distribute the route itself.

This can be useful in the following situations:

- If two immediately adjacent Label Switched Routers (LSRs) are also BGP peers, then label distribution can be done without the need for any other label distribution protocol.

- Suppose one's network consists of two "classes" of LSR: exterior LSRs, which interface to other networks, and interior LSRs, which serve only to carry traffic between exterior LSRs. Suppose that the exterior LSRs are BGP speakers. If the BGP speakers distribute MPLS labels to each other along with each route they distribute, then as long as the interior routers support MPLS, they need not receive any of the BGP routes from the BGP speakers.

If exterior router A needs to send a packet to destination D, and $A^{\prime} S$ BGP next hop for $D$ is exterior router $B$, and $B$ has mapped label L to D, then A first pushes L onto the packet's label stack. A then consults its IGP to find the next hop to B, call it C. If $C$ has distributed to A an MPLS label for the route to B, A can push this label on the packet's label stack, and then send the packet to $\mathrm{C}$.

If a set of BGP speakers are exchanging routes via a Route Reflector [BGP-RR], then by piggybacking the label distribution on the route distribution, one is able to use the Route Reflector to distribute the labels as well. This improves scalability quite significantly. Note that if the Route Reflector is not in the forwarding path, it need not even be capable of forwarding MPLS packets.

Label distribution can be piggybacked in the BGP Update message by using the BGP-4 Multiprotocol Extensions attribute [RFC 2283]. The label is encoded into the NLRI field of the attribute, and the SAFI 
("Subsequent Address Family Identifier") field is used to indicate that the NLRI contains a label. A BGP speaker may not use BGP to send labels to a particular BGP peer unless that peer indicates, through BGP Capability Advertisement, that it can process Update messages with the specified SAFI field.

3. Carrying Label Mapping Information

Label mapping information is carried as part of the Network Layer Reachability Information (NLRI) in the Multiprotocol Extensions attributes. The AFI indicates, as usual, the address family of the associated route. The fact that the NLRI contains a label is indicated by using SAFI value 4 .

The Network Layer Reachability information is encoded as one or more triples of the form <length, label, prefix>, whose fields are described below:

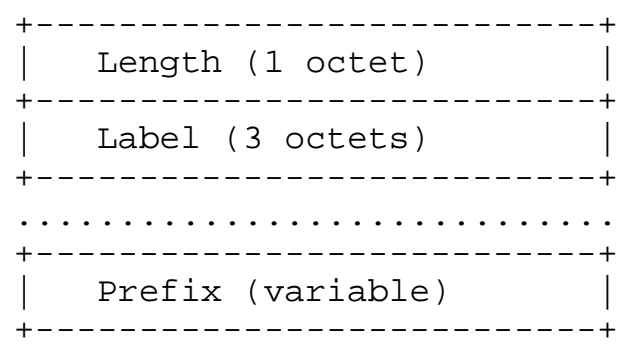

The use and the meaning of these fields are as follows:

a) Length:

The Length field indicates the length in bits of the address prefix plus the label(s).

b) Label :

The Label field carries one or more labels (that corresponds to the stack of labels [MPLS-ENCAPS]). Each label is encoded as 3 octets, where the high-order 20 bits contain the label value, and the low order bit contains "Bottom of Stack" (as defined in [MPLS-ENCAPS ] ) .

c) Prefix:

The Prefix field contains address prefixes followed by enough trailing bits to make the end of the field fall on an octet boundary. Note that the value of trailing bits is irrelevant. 
The label(s) specified for a particular route (and associated with its address prefix) must be assigned by the LSR which is identified by the value of the Next Hop attribute of the route.

When a BGP speaker redistributes a route, the label(s) assigned to that route must not be changed (except by omission), unless the speaker changes the value of the Next Hop attribute of the route.

A BGP speaker can withdraw a previously advertised route (as well as the binding between this route and a label) by either (a) advertising a new route (and a label) with the same NLRI as the previously advertised route, or (b) listing the NLRI of the previously advertised route in the Withdrawn Routes field of an Update message. The label information carried (as part of NLRI) in the Withdrawn Routes field should be set to 0x800000. (Of course, terminating the BGP session also withdraws all the previously advertised routes.)

4. Advertising Multiple Routes to a Destination

A BGP speaker may maintain (and advertise to its peers) more than one route to a given destination, as long as each such route has its own label (s).

The encoding described above allows a single BGP Update message to carry multiple routes, each with its own label(s).

In the case where a BGP speaker advertises multiple routes to a destination, if a route is withdrawn, and a label(s) is specified at the time of withdrawal, only the corresponding route with the corresponding label is withdrawn. If a route is withdrawn, and no label is specified at the time of withdrawal, then only the corresponding unlabeled route is withdrawn; the labeled routes are left in place.

5. Capability Advertisement

A BGP speaker that uses Multiprotocol Extensions to carry label mapping information should use the Capabilities Optional Parameter, as defined in [BGP-CAP], to inform its peers about this capability. The MP_EXT Capability Code, as defined in [BGP-MP], is used to advertise the (AFI, SAFI) pairs available on a particular connection.

A BGP speaker should not advertise this capability to another BGP speaker unless there is a Label Switched Path (LSP) between the two speakers. 
A BGP speaker that is capable of handling multiple routes to a destination (as described above) should use the Capabilities Optional Parameter, as defined in [BGP-CAP], to inform its peers about this capability. The value of this capability is 4.

6. When the BGP Peers are not Directly Adjacent

Consider the following LSR topology: A--B--C--D. Suppose that D distributes a label L to A. In this topology, A cannot simply push L onto a packet's label stack, and then send the resulting packet to $B$. $D$ must be the only LSR that sees $L$ at the top of the stack. Before A sends the packet to $B$, it must push on another label, which was distributed by B. B must replace this label with yet another label, which was distributed by $C$. In other words, there must be an LSP between $A$ and D. If there is no such LSP, A cannot make use of label L. This is true any time labels are distributed between non-adjacent LSRs, whether that distribution is done by BGP or by some other method.

This document does NOT specify any procedure for ensuring in real time that label distribution between non-adjacent LSRs is done only when the appropriate MPLS infrastructure exists in the network or networks connecting the two LSRs. Ensuring that the proper infrastructure exists is an issue for network management and operation.

7. Security Considerations

When an LSR A is directly connected to an LSR B via a point-to-point interface, then when A receives packets over that interface, it knows that they come from B. This makes it easy for A to discard any packets from B whose top labels are not among the labels that A distributed to B. That is, A can easily ensure that B only uses those labels which it is entitled to use. This technique can be used to prevent "label spoofing", i.e., the situation in which an LSR imposes a label which has not been properly distributed to it.

The procedures discussed in this document would commonly be used when the label distribution peers are separated not merely by a point-topoint link, but by an MPLS network. This means that when an LSR A processes a labeled packet, it really has no way to determine which other LSR B pushed on the top label. Hence it cannot tell whether the label is one which $B$ is entitled to use. In fact, when Route Reflectors are in use, A may not even know the set of LSRs which receive its label mappings. So the previous paragraph's technique for preventing label spoofing does not apply. 
It is possible though to use other techniques to avoid label spoofing problems. If, for example, one never accepts labeled packets from the network's "external" interfaces, and all the BGP-distributed labels are advertised via IBGP, then there is no way for an untrusted router to put a labeled packet into the network. One can generally assume that one's IBGP peers (or the IBGP peers of one's Route Reflector) will not attempt label spoofing, since they are all under the control of a single administration.

This condition can actually be weakened significantly. One doesn't need to refuse to accept all labeled packets from external interfaces. One just needs to make sure that any labeled packet received on an external interface has a top label which was actually distributed out that interface.

Then a label spoofing problem would only exist if there are both trusted and untrusted systems out the same interface. One way to avoid this problem is simply to avoid this situation.

8. Acknowledgments

Thanks to Ravi Chandra, Enke Chen, Srihari Ramachandra, Eric Gray and Liam Casey for their comments.

9. References

[BGP-4] Rekhter, Y. and T. Li, "A Border Gateway Protocol 4 (BGP-4) ", RFC 1771, March 1995.

[BGP-CAP] Chandra, R. and J. Scudder, "Capabilities Advertisement with BGP-4", RFC 2842, May 2000 .

[BGP-MP] Bates, T., Rekhter, Y, Chandra, R. and D. Katz, "Multiprotocol Extensions for BGP-4", RFC 2858, June 2000 .

[BGP-RR] Bates, T. and R. Chandra, "BGP Route Reflection: An alternative to full mesh IBGP", RFC 1966, June 1996.

[MPLS-ARCH] Rosen, E., Vishwanathan, A. and R. Callon, "Multiprotocol Label Switching Architecture" RFC 3031 , January 2001 .

[MPLS-ENCAPS] Rosen, E., Tappan, D., Fedorkow, G., Rekhter, Y., Farinacci, D., Li, T. and A. Conta, "MPLS Label Stack Encoding", RFC 3032, January 2001. 
10. Authors' Addresses

Yakov Rekhter Juniper Networks

1194 N. Mathilda Avenue

Sunnyvale, CA 94089

EMail: yakov@juniper.net

Eric Rosen

Cisco Systems, Inc.

250 Apollo Drive

Chelmsford, MA 01824

EMail: erosen@cisco.com 
11. Full Copyright statement

Copyright (C) The Internet Society (2001). All Rights Reserved.

This document and translations of it may be copied and furnished to others, and derivative works that comment on or otherwise explain it or assist in its implementation may be prepared, copied, published and distributed, in whole or in part, without restriction of any kind, provided that the above copyright notice and this paragraph are included on all such copies and derivative works. However, this document itself may not be modified in any way, such as by removing the copyright notice or references to the Internet society or other Internet organizations, except as needed for the purpose of developing Internet standards in which case the procedures for copyrights defined in the Internet Standards process must be followed, or as required to translate it into languages other than English.

The limited permissions granted above are perpetual and will not be revoked by the Internet society or its successors or assigns.

This document and the information contained herein is provided on an "AS IS" basis and THE INTERNET SOCIETY AND THE INTERNET ENGINEERING TASK FORCE DISCLAIMS ALL WARRANTIES, EXPRESS OR IMPLIED, INCLUDING BUT NOT LIMITED TO ANY WARRANTY THAT THE USE OF THE INFORMATION HEREIN WILL NOT INFRINGE ANY RIGHTS OR ANY IMPLIED WARRANTIES OF MERCHANTABILITY OR FITNESS FOR A PARTICULAR PURPOSE.

Acknowledgement

Funding for the RFC Editor function is currently provided by the Internet society. 